\title{
Read, Play and Learn: An Interactive E-book for Children with Autism
}

\author{
Vedad Hulusic ${ }^{1}$ and Nirvana Pistoljevic ${ }^{2}$ \\ 1 University Sarajevo School of Science and Technology, Hrasnicka cesta 3a, Sarajevo \\ 71000, Bosnia and Herzegovina \\ 2 Teachers College, Columbia University, 525 West 120th St., New York NY 10027, \\ United States of America
}

\begin{abstract}
Serious games can easily engage students, captivate and maintain their attention allowing them both learning with an adult, or on their own. But, teaching children with developmental disabilities like ASD, requires special set of tools and methods, due to decreased level of attention towards stimuli presented and lessened capability to learn in ways typical children do. Interactive multisensory computer based instruction seems to be a good match for these diverse learners because it offers multisensory learning experience, interactive practice with constant feedback, increased learning opportunities, and customization to each child's needs. In this paper we present a web-based interactive educational e-book designed to engage a learner with additional auditory-visual stimulation related to the text being read in two languages, and by providing multi layered questions about the story read, for comprehension. Our educational goals were to teach children novel vocabulary, counting, identifying numbers and colors, and responding to inference questions.
\end{abstract}

Keywords: children, autism, interactive e-book, learn unit, education

\section{Introduction}

Autism Spectrum Disorder (ASD) is a neurodevelopmental disorder that is detectable or apparent early in development, before age 2 [20], and its characterized by the lack of language development, socialization and development of patterns of rigid, repetitive, auto-stimulating behaviors that interfere with overall functioning of a person [3]. Children with ASD often have difficulty processing sensory information, by under- or over-processing different inputs, which in turn affects their learning. Studies have shown that children with autism may master more educational objectives using a computer compared to traditional teaching strategies, and are more interested and motivated to learn through computer-assisted instruction [7,34]. Price compared comprehension and information acquisition across two conditions: reading interactive e-books (on iPads) vs. printed books with 30 students diagnosed with ASD, and found positive outcomes for each participant using e-books [23]. In addition, teachers' survey reveled that the students found iPads motivating, which made them reinforcers, and in turn decreased their inappropriate behaviors in the classroom. 
For over 50 years, research showed that the basic teaching unit, upon which all learning and teaching are based on is a three-term-contingency (i.e. a learn unit) $[27,15,16]$. A learn unit is an observable and measurable interaction between a teacher and a learner, and it consists of two or more antecedent-behaviorconsequence contingencies. A-antecedent is a stimulus/stimuli presented by a teacher or a computer/teaching device (i.e. visual stimuli - picture of a bird and an auditory stimuli - find a bird); B-behavior is a response to an antecedent (i.e. student touching a bird on the screen); and a C-consequence is delivered in a way of reinforcement for correct responses and correction for an incorrect responses. Corrections for incorrect response guide the student to emit a correct response, in order not to demotivate a learner and to provide him/her with a learning opportunity. Skinner's tree-term-contingency has been proven effective not only as a teacher-student interaction, but as one between a learner and a teaching device, famous Skinner's teaching machine [27,28] or Emurian et al. 's computerized instruction [10]. Therefore, a serious game we have designed and developed, is entirely based on the learn unit, an interlocking three-term contingency between a computer that presents visual and auditory antecedents and delivers feedback, and a student who emits the responses to the computer's antecedents [18].

Serious games have become an effective tool in treatment of various physical and psychological limitations, as well as education of diverse populations. Using this medium users are exposed to deeply engaging, visually dynamic, rapidly paced, and highly satisfying experiences, in comparison to conventional teaching methods and therapies [13]. In addition, serious gaming has contributed to several fields, such as education [9], simulation [25], health [2], cultural heritage [1] and others.

Serious games have been used for medical applications, including treatment of ASD [33], and can be divided into educational and treatment games. Each of these categories can be further divided into subcategories [22]. Existing games, focusing on treatment of social interaction with children with ASD, are used to reinforce emotion recognition [26], facial recognition [30], and foster collaboration through computer gaming $[4,5]$. Noor et al. reviewed articles from 2011 and 2012, putting the focus on technology and serious games classification, as well as on the developed serious games for treating children with ASD and/or for education [22]. Another review by Zakari et al., considering articles from 2004 to 2014, divided them across several criteria: technology platform, 2D/3D, learning objectives and type of interaction [35]. According to these classifications, our game could be defined as a tablet/desktop/laptop, 2D, touch-screen for learning counting 1-10, identifying letters, colors and objects, and understanding the semantics of the scene and narration.

\section{The life of "The Wolf in Love"}

"The Wolf in Love" e-book has two ancestors: an inclusive theatre play and an interactive tactile (hard copy) children's book. 


\subsection{The theatre play}

The theatre play was initiated by EDUS-Education for All, a non-for-profit organization that advances education for children with and without disabilities in Bosnia and Herzegovina in order to create an opportunity for children with ASD to engage in creative activity with typically developing peers. Marija FeketeSullivan wrote a screen play and the children's book storyline [12]. The play was then created in co-operation with the Aparat Thetre and SARTR (Sarajevo War Theatre), and has been performed several times by children actors with and without developmental disorders, Figure 1.
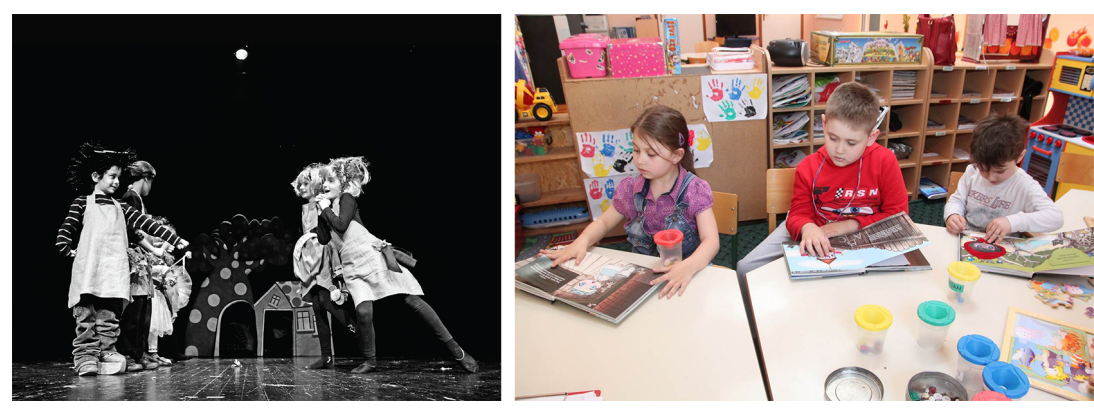

Fig. 1. A scene from the theatre play (left) and the children interaction with the printed book (right).

\subsection{The hard copy}

Following the success of the play and children's enjoyment, which was mainly a result of the idea of respecting the differences and ignoring false boundaries between children, a children book with tactile stimulations and interactive elements (pulling, flipping, texture changes, and similar) was designed and printed with the support of UNICEF-BH, Figure 1. The book was aimed to stimulate and reinforce comprehensive development and school readiness skills as the main precondition for realizing the full individual potential of a child. It was designed to provide stimulation for 3 to 12 year-old children with and without disabilities, especially focusing on the multisensory learning needs of children with ASD. The book was also bilingual (Bosnian-Croatian-Serbian and English languages), and each page had options for discovery learning and tactile sensations in order to draw and keep children's attention. For younger learners or children with disabilities, each page provided learning not only through auditory stimulation of someone reading to them, but through full sensory exploration learning per each page as well (i.e. texture changes, manipulative parts of a page, strong colors and salient target characters, letters, numbers, etc.). Older learners were able to read the story but also to learn words in a second language since on each page, text was printed in both BHS and English languages as direct translations. These objectives were all aligned with the $\mathrm{BH}$ educational curriculum. 


\subsection{The e-book}

According to the UNICEF-BH MICS Survey, only $56 \%$ of children in Bosnia and Herzegovina, ages birth through 5 years-old, have at least 3 children's books in the house [31]. However, we are aware of the trends in the world today where media dominate children's lives through watching TV, playing video games, reading stories through e-readers, tablets, and smartphones. In the USA, the percent of children with access to some type of smart mobile device at home (e.g. smartphone, tablet) has jumped from 52\% to $75 \%$ from 2011 to 2013 [17]. Seventy-two percent of children ages 0-8 use a mobile device for some type of media activity such as playing games (63\% of the time), watching videos, using apps, or reading books (only $30 \%$ of the time) [17]. Video games in particular provide an ideal multimedia tool that presents concepts in a way that are engaging, informal, and fun for children [24]. Therefore, a potential benefit of educational video game is the value of generating engagement and motivation to learn for a student [6]. New multimedia and interactive features of the animated electronic storybooks were found superior when it comes to vocabulary acquisition in kindergartens compared to static e-book version of the same text in Netherlands [29]. Also, eye-tracking studies have demonstrated that children fixate longer on details in illustrations that are highlighted in the text [32]. Glenburg and Langston demonstrated that comprehension is better when the text is followed by illustration as pictures help to build mental models [14].

Guided by all these findings, we have designed our interactive e-book to attract attention of a learner, to engage him/her by features additional to the text being read in two languages (i.e. auditory stimulation when clicked on a certain object in the scene: naming the object or making a related sound) and by providing a multi layered question about the scene or story read. The questions on each page increase in difficulty to accommodate different levels of learners (young or a child with a disability vs. older typically developing child), Table 1.

\begin{tabular}{|c|c|}
\hline Question & Educational goal \\
\hline \hline 1 & $\begin{array}{r}\text { Selection-based question always with varied antecedents } \\
\text { (i.e. "point to", "find", "where is", "touch") }\end{array}$ \\
\hline 2 & $1: 1$ correspondence question, teaching children to count objects \\
\hline 3 & $\begin{array}{r}\text { Production responding, an inference question, where the child reads a } \\
\text { short text and makes conclusions based on the comprehension of the story }\end{array}$ \\
\hline
\end{tabular}

Table 1. The educational goals were to teach children to identify target objects (i.e. learn the vocabulary), 1:1 correspondence or learning to count objects from 1 to 10, to identify numbers 1-5, identify colors, and respond to inference questions.

One of the goals of both the entertainment and serious games is adaptivity and dynamic content generation $[8,19]$, in order to efficiently balance the game flow and thus avoid frustration and/or boredom. In our game this is achieved by allowing the user to skip to the next page without being forced to answer the higher-level questions. In addition, the order of predefined answers is randomized and multiple target stimuli for "point to" type of questions are provided. 
In addition, the cartoon-like, low-fidelity graphics in our game is used for several reasons. The first one is that the same graphics has been used in the printed book. As this is an extension we wanted to preserve the same visual appearance. Secondly, the targeted population for our game is used to this graphics style from various children books and cartoons. Finally, we wanted to avoid the "Uncanny valley" phenomenon [21], in which a high-fidelity human-like computer-generated character is less preferred than its cartoon-like counterpart.

Our overall goal was to enrich the book reading experience for different levels of readers and to provide an engaging learning environment for children with disabilities, especially multisensory needs of children with ASD. The game can be accessed at http://edusbih.org/book/.

\section{E-book Development}

The e-book was developed using several web technologies: HTML5, CSS3, JavaScript, jQuery and AJAX. These technologies allow for easy-to-use, real-time interaction through the web-based front end.
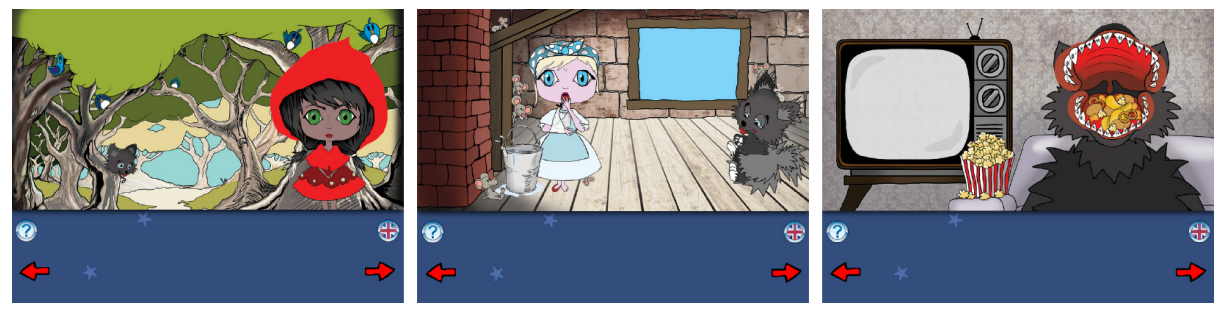

Fig. 2. Three scenes from the e-book: scene 2 (left), scene 5 (middle) and scene 10 (right).

In the web implementation of the e-book, the pages were called scenes. There are 13 scenes in total, named scene1.html through scene13.html, Figure 2. By default, when the e-book is started, scene1.html is loaded. All scenes are displayed through a single file, index.html. This is achieved through a navigation controller that loads a requested scene by switching the current html content with the content of the corresponding scene through AJAX, Figure 3. At the same time, JavaScript method loadScene, defined within index.html, resets the global variables was_corrected - used to indicate the correction; and questions_active indicating if the questions were triggered. At this point the event listeners take over and wait for the user to interact with the game.

All html files have event listeners which are responsible for the page interaction. index.html controls the logic for the questions and answers, the language selection and for scene traversal, Figure 4. Individual scene files are responsible for the events at each particular scene, e.g. playing the sounds, hiding and showing the elements, selecting the questions, and initiating the corrections. 


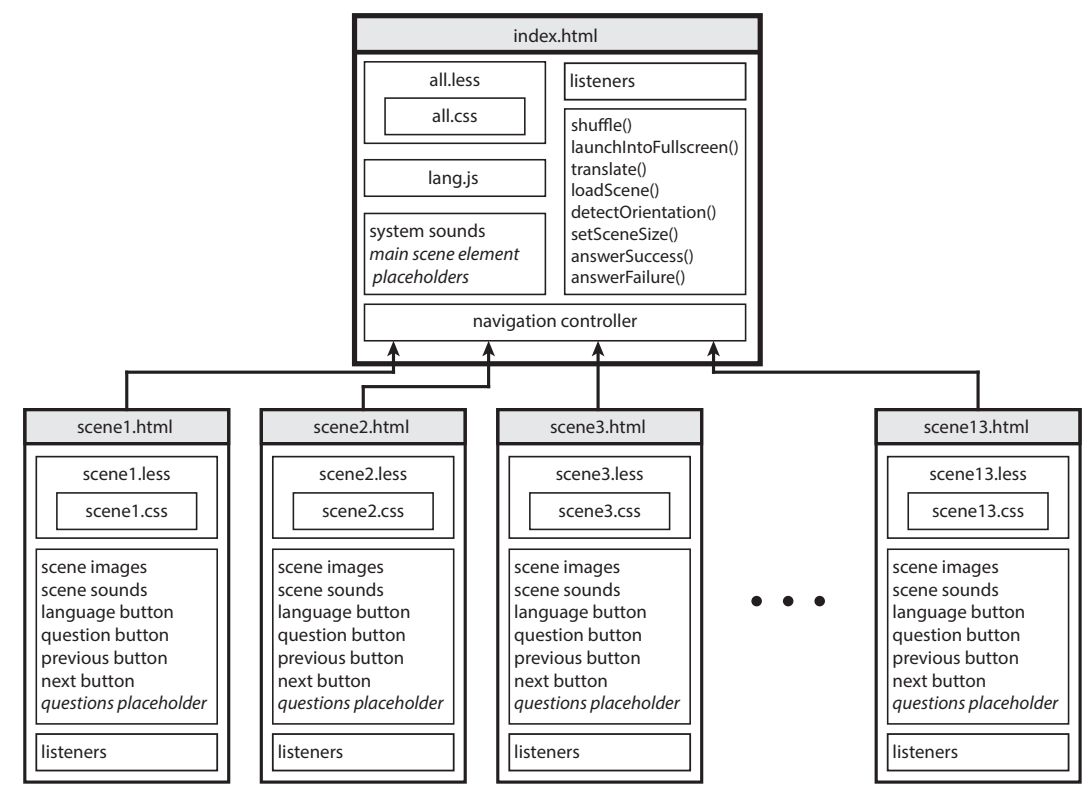

Fig. 3. A schematic view of the game structure.

All the styling, including visual appearance, sizes, positions and effects, is done using Less elements preprocessor [11]. Namely, each scene has its own .less file which defines the above-mentioned features for that page. On top of that, there is an all.less file which defines the general page appearance including the language and question bar (Figure 4), responsiveness, correction and animations.

Everything in the application that can be translated has its key in the lang.js file. By clicking on the language selection button, the method translate() is called. The method translates all the system and local strings that are defined for the corresponding scene. The language can be selected at the opening scene, and also toggled throughout the game. This is an important feature that fosters learning a foreign language.

Each scene contains three question, two of which have three provided answers. For each such question only one of them, within its html element of the class answer, has class correct assigned. Questions that require interaction with the active scene elements, i.e. that use the "where is", "point to" or similar antecedent, have their own logic implemented within their belonging scene. This logic is based on an additional listener that checks if the touched element has a class correct assigned to it. If so, the answer is considered as correct. For each correct answer the "applause" sound is played and one of the text responses ("Great job!", "Excellent!", "That's correct!") is randomly provided. In case of the incorrect answer the correction is invoked. All the provided answers are shuffled using the method shuffle() in the index.html file.

The correction for the incorrect answers is provided by either adding the blinking yellow frame to the correct answer (Figure 4) for the questions with the 


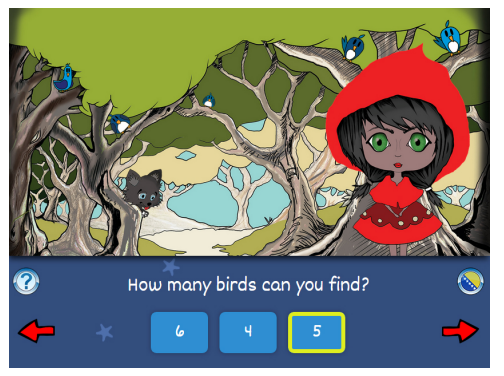

Fig. 4. A correction indicating the correct answer. Bottom box: the question button (top left), the language button (top right), the question and answers (middle), navigational buttons (bottom left and right).

provided answers, or by adding a red halo around the correct object (Figure 5) for the questions with the "pointing to" antecedent.
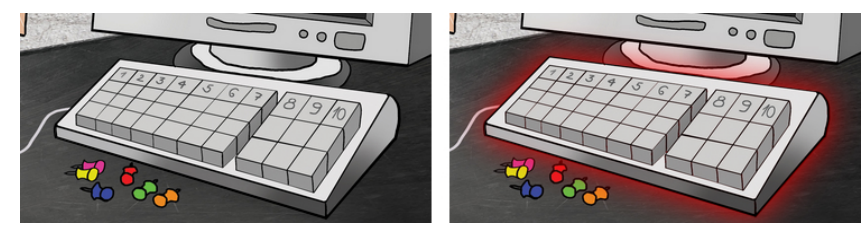

Fig. 5. Correction invoked after an element other than the keyboard had been touched.

In each scene there are a number of interactive elements. These elements, when touched, either make corresponding sound, such as tweeting for bird or howling for wolf, or are being named in the selected language. At the same time, they are either smoothly scaled up and down, so they gently pop up, or animated so they move across the scene. This is achieved by assigning the scaleanimation class to the corresponding element, and defining the animation code in the corresponding .less file.

Finally, each scene has a bilingual narration, which is part of the storyline that introduces the child to the current scene. It is triggered by either clicking on the narrating character if in the scene (Figure 6), or on any non-interactive element in the scene if the narrator is not present. Once triggered, it is both displayed visually on a predefined spot and played audibly. All the voice overs and the object naming audio files have been narrated by children.

\section{Conclusions and Future Work}

Our interactive e-book provides a multisensory experience to relate basic learning concepts to children through game-like experience. Children can hear the text being narrated in one of the two languages of their choice, they can read along and practice reading skills or just relay on visual stimuli and textually respond to it. They can engage in a game to explore each page of the book or for younger 

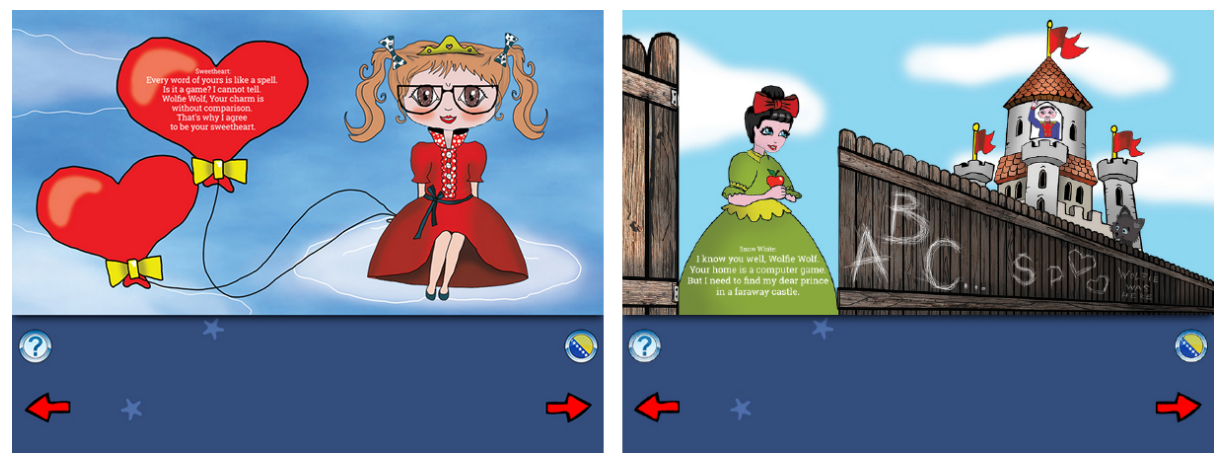

Fig. 6. The visual appearance of the narration once triggered.

users, just the interactivity and animations on each page are something to retain their attention. It is a multiuse, multilevel educational interactive application of the latest research in education, interactive e-books and serious games.

Since this is a pilot project, the e-book has only been preliminary tested with several children with and without developmental delays, in order to eliminate any potential technical malfunctioning. Nonetheless, it showed an indication of a great potential, as all children seemed to enjoy using it and expressed high interest in reusing it. The next step is to test the e-book and its interactive features on the acquisition of the novel vocabulary, foreign language vocabulary, 1:1 correspondence (i.e. counting), colors, and comprehension of the story. We would like to compare the effect and the experiences of children with disabilities with typically developing children.

On the other side, there were many considerations and limitations on the technical side of the game development, which could be overcome in the future and allow for further technical enhancement of the game. One such consideration was the scene transition. A simple transition, where the internet connection is over $1 \mathrm{Mbps}$, has been straightforward to implement and it is rendered smoothly without any delays. However, for lower bandwidths, which are not uncommon in this region, and also for mobile connections (3G and similar), we implemented the background scene loading that allows the user to interact with the current scene while the next one is being loaded. Once the requested scene is loaded, the transition is made.

One of the main limitations in the development was the input graphics that we had from the designing team of the printed book. Namely, all the graphics were raster, and only partially layered, which made it impossible to extract many scene objects from the background without major interventions on the drawings. Furthermore, it restricted the game resolution and object animations. In the future versions of the e-book we will make more scene object interactive and potentially improve the object animations. In addition, we will make the game more responsive so that it fits all the screen size factors and ratios more naturally. 
Acknowledgments. The authors would like to thank employees of the non-forprofit organization EDUS-Education for All. We would also like to thank Bakir Husovic and Namik Mesic for helping with the game development. Finally, we would like to thank everyone who was involved and supported the development of the theatre play, printed book and the e-book. The pilot project was funded by the UNICEF-BH.

\section{References}

1. Anderson, E.F., McLoughlin, L., Liarokapis, F., Peters, C., Petridis, P., de Freitas, S.: Developing serious games for cultural heritage: a state-of-the-art review. Virtual reality $14(4), 255-275(2010)$

2. Arnab, S., Dunwell, I., Debattista, K.: Serious games for healthcare: Applications and implications pp. 1-370 (2013)

3. Association, A.P., et al.: Diagnostic and Statistical Manual of Mental Disorders (DSM-5®). American Psychiatric Pub (2013)

4. Battocchi, A., Gal, E., Sasson, A.B., Pianesi, F., Venuti, P., Zancanaro, M., Weiss, P.: Collaborative puzzle game-an interface for studying collaboration and social interaction for children who are typically developed or who have autistic spectrum disorder. Proc. 7th ICDVRAT with ArtAbilitation pp. 127-134 (2008)

5. Battocchi, A., Pianesi, F., Venuti, P., Ben-Sasson, A., Gal, E., Weiss, P.: Collaborative puzzle game: Fostering collaboration in children with autistic spectrum disorder (asd) and with typical development. In: 2009 Virtual Rehabilitation International Conference (2009)

6. Bourgonjon, J., Valcke, M., Soetaert, R., Schellens, T.: Students perceptions about the use of video games in the classroom. Computers \& Education 54(4), 1145-1156 (2010)

7. Chen, S.H., Bernard-Opitz, V.: Comparison of personal and computer-assisted instruction for children with autism. Mental Retardation 31(6), 368-376 (1993)

8. Correa, O., Cuervo, C., Perez, P., Arias, A.: A new approach for self adaptive video game for rehabilitation-experiences in the amblyopia treatment. In: Serious Games and Applications for Health (SeGAH), 2014 IEEE 3rd International Conference on. pp. 1-5. IEEE (2014)

9. De Freitas, S.I.: Using games and simulations for supporting learning. Learning, media and technology 31(4), 343-358 (2006)

10. Emurian, H., Hu, X., Wang, J., Durham, A.: Learning java: a programmed instruction approach using applets. Computers in Human Behavior 16(4), $395-422$ (2000)

11. Fadeyev, D.: Less elements. http://lesselements.com/

12. Fekete-Sullivan, M.: Zaljubljeni vuk / The Wolf in Love. EDUS (2015)

13. Foreman, J.: Next-generation educational technology versus the lecture. EDUCAUSE Review Magazine 38(4), 12-22 (2003)

14. Glenberg, A.M., Langston, W.E.: Comprehension of illustrated text: Pictures help to build mental models. Journal of memory and language 31(2), 129-151 (1992)

15. Greer, R.D.: The teacher as a strategic scientist: A solution to our education crisis? Behavior and Social Issues 1(2), 25-41 (1991)

16. Greer, R.D.: Designing teaching strategies: An applied behavior analysis system approach. Academic Press (2002) 
17. Holloway, D., Green, L., Livingstone, S.: Zero to eight: Young children and their internet use (2013)

18. Hulusic, V., Pistoljevic, N.: "lefca": Learning framework for children with autism. Procedia Computer Science 15, 4-16 (2012)

19. Lopes, R., Bidarra, R.: Adaptivity challenges in games and simulations: a survey. Computational Intelligence and AI in Games, IEEE Transactions on 3(2), 85-99 (2011)

20. Luyster, R., Richler, J., Risi, S., Hsu, W.L., Dawson, G., Bernier, R., Dunn, M., Hepburn, S., Hyman, S.L., McMahon, W.M., et al.: Early regression in social communication in autism spectrum disorders: a cpea study. Developmental neuropsychology 27(3), 311-336 (2005)

21. Mori, M., MacDorman, K.F., Kageki, N.: The uncanny valley [from the field]. Robotics \& Automation Magazine, IEEE 19(2), 98-100 (2012)

22. Noor, M., Shahbodin, F., Pee, C., et al.: Serious game for autism children: review of literature (2012)

23. Price, A.: Making a difference with smart tablets. Teacher Librarian 39(1), 31-34 (2011)

24. Rieber, L.P., Noah, D.: Games, simulations, and visual metaphors in education: antagonism between enjoyment and learning. Educational Media International 45(2), 77-92 (2008)

25. Shelton, B.E., Hedley, N.R.: Using augmented reality for teaching earth-sun relationships to undergraduate geography students. In: Augmented Reality Toolkit, The First IEEE International Workshop. pp. 8-pp. IEEE (2002)

26. Silver, M., Oakes, P.: Evaluation of a new computer intervention to teach people with autism or asperger syndrome to recognize and predict emotions in others. Autism 5(3), 299-316 (2001)

27. Skinner, B.F.: Teaching machines. Science 128(3330), 969-977 (1958)

28. Skinner, B.F.: The Technology of Teaching. Appleton-Century-Crofts (1968)

29. Smeets, D., Bus, A.: The interactive animated e-book as a word learning device for kindergartners. Applied Psycholinguistics 22(1), 1-22 (2014)

30. Tanaka, J.W., Wolf, J.M., Klaiman, C., Koenig, K., Cockburn, J., Herlihy, L., Brown, C., Stahl, S., Kaiser, M.D., Schultz, R.T.: Using computerized games to teach face recognition skills to children with autism spectrum disorder: The lets face it! program. Journal of Child Psychology and Psychiatry 51(8), 944-952 (2010)

31. UNICEF-BH: Bosnia and Herzegovina Multiple Indicator Cluster Survey (MICS) 20112012. UNICEF-BH (2013)

32. Verhallen, M.J., Bus, A.G.: Young second language learners visual attention to illustrations in storybooks. Journal of Early Childhood Literacy 11(4), 480-500 (2011)

33. Wang, Q., Sourina, O., Nguyen, M.K.: Eeg-based" serious" games design for medical applications. In: Cyberworlds (CW), 2010 International Conference on. pp. 270-276. IEEE (2010)

34. Whalen, C., Massaro, D.W., Franke, L.: Generalization in computer-assisted intervention for children with autism spectrum disorders. In: Real Life, Real Progress for Children with Autism Spectrum Disorders, pp. 223-233. Brookes Publishing Co. (2009)

35. Zakari, H., Ma, M., Simmons, D.: A review of serious games for children with autism spectrum disorders (asd). In: Ma, M., Oliveira, M., Baalsrud Hauge, J. (eds.) Serious Games Development and Applications, Lecture Notes in Computer Science, vol. 8778, pp. 93-106. Springer International Publishing (2014), http://dx.doi.org/10.1007/978-3-319-11623-5-9 